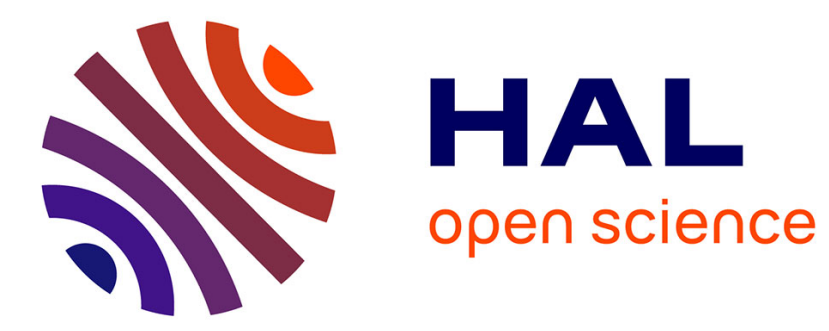

\title{
La protection des rivières et des lacs contre la pollution
}

Paul Vivier

\section{To cite this version:}

Paul Vivier. La protection des rivières et des lacs contre la pollution. Revue forestière française, 1959,

7, pp.493-502. 10.4267/2042/27520 . hal-03382229

\section{HAL Id: hal-03382229 \\ https://hal.science/hal-03382229}

Submitted on 18 Oct 2021

HAL is a multi-disciplinary open access archive for the deposit and dissemination of scientific research documents, whether they are published or not. The documents may come from teaching and research institutions in France or abroad, or from public or private research centers.
L'archive ouverte pluridisciplinaire HAL, est destinée au dépôt et à la diffusion de documents scientifiques de niveau recherche, publiés ou non, émanant des établissements d'enseignement et de recherche français ou étrangers, des laboratoires publics ou privés. 


\section{REVUE FORESTIERE FRANCCAISE}

I959 Julllet $\mathrm{N}^{\circ} 7$

Dans ce numéro: P. Vivier: La protection des rivières et des lacs contre: la pollution. - J.-P.-A. Debreyne: Un élément indispensable et méconnu des régénérations en essences forestières de valeur: la faune des carnassiers et des rapaces. L'exemple des forêts domaniales du Valois. - G. Estager: Les ventes d'automne de coupes de bois sur pied de l'exercice 1958 dans les forêts soumises au régime forestier. - $\mathrm{H}$. Gaussen: Un nouveau genre de conifères: Cathaya. - J. Bossavy: Evacuation des eaux sur les routes forestières. Un nouveau matériel.

\section{LA PROTECTION DES RIVIẼRES ET DES LACS CONTRE LA POLLUTION

Le problème de la pollution des eaux par les rejets d'eaux industrielles ou urbaines est ancien. Dès l'apparition de l'industrie, au début du siècle dernier, il préoccupait les pouvoirs publics et, dans un rapport officiel adressé au Ministre du Commerce en I850, sur le repeuplement des cours d'eau, le naturaliste bien connu Henri Milne-EDwards, membre de l'Institut, écrivait: “On a remarqué aussi que les eaux provenant des usines à papier qui contiennent une assez grande quantité de chlore provenant du blanchiment des chiffons sont nuisibles aux poissons. C'est là une cause de destruction clont il est juste de tenir' compte )" (2).

Avec l'extension de l'industrie et l'accroissement de la population urbaine, le problème de la pollution a pris, en France, comme dans le Monde, une importance considérable. Ce n'est pas seulement le poisson qui est tué ou incommodé par les déversements d'eau polluée, mais l'état biologique du milieu récepteur se trouve souvent profondément altéré ; les riverains ne sont pas sans en souffrir : mauvaises odeurs, baignades impossibles; enfin le tourisme est lui-même parfois compromis, comme nous le verrons tout à l'heure.

Les poissons sont, en effet, extrêmement sensibles aux toxiques.

Le temps au bout duquel le poisson meurt (mort apparente) ou

(I) Conférence faite le 20 mai I959 à l'Ecole Nationale d'Administration, à l'occasion de la $10^{\circ}$ rencontre organisée par le Comité d'Administration $\mathrm{Pu}$ blique de 1'Union de l'Europe occidentale.

(2) Rapport sur l'empoissonnement des rivières reproduit par HAxo. De la fécondation artificielle des oufs de poissons et de leur éclosion. Epinal, 1853, I, 93. 
du moins conmence à souffrir sérieusement du poison au point de perdre l'équilibre, appelé temps de manifestation du poison ou temps de survie est lié à la concentration du produit par une équation de la forme $\mathrm{X}^{\mathrm{n}} \mathrm{Y}=\mathrm{K}$. dont la représentation graphique a l'allure d'une hyperbole. On constate, en effet, que pour de faibles concentrations d'un poison donné, le temps de manifestation est très long et se rapproche de l'infini pour une limite de concentration déterminée pour laquelle le poison est sans effet grave sur l'organisme; il y a ainsi un seuil de concentration. Le temps d'action du toxique diminue l'abord très rapidement, à mesure que l'on dépasse le seuil de concentration, puis les teneurs augmentant, le temps de manifestation diminue, mais de plus en plus lentement, pour se rapprocher d'une valeur constante minima atteinte pour des concentrations très élevées. Tant qu'on expérimente dans des limites de concentrations raisonnables du point de vue physiologique, on constate qu'il existe aussi un seuil de temps de manifestation de l'empoisonnement, comme il existe un seuil de concertration. Mais ces notions ont surtout une valeur théorique.

En pratique, pendant un temps donné, les deux doses sont particulièrement intéressantes à connaitre: c'est-à-dire la plus petite dose de toxique capable d'entraîner la mort du poisson (dose minima mortelle) et la dilution limite, c'est-à-dire la plus forte dose de poison que le sujet peut supporter sans manifester de fatigue. Ces doses sont variables suivant les espèces de poisson, leur âge, le titre alcalimétrique de l'eau, la température, la dose d'oxygène dissous et naturellement suivant le tcmps d'observation. Ces doses sont cependant très faibles. Il suffit de 4 à $6 \mathrm{mg}$ d'acide chlorhydrique pour tuer un Vairon en to heures et une dose dix fois plus faible de cyanure de Potassium pour tuer une truitelle. I1 ne saurait être question ici de donner les indications sur la façon de procéder à ces tests toxicologiques dont une commission internationale officieuse comprenant cles représentants de la Suisse, de la Belgique et de la France a défini les conditions opératoires à Zurich, en 1953. Mais ces conditions scientifiques rigoureuses demandent beaucoup de temps et ne peuvent être employées que pour des travaux de recherches.

En pratique, les tests se font dans le cours d'eau pollué lui-même, au moyen de cages grillagées où sont enfermés les poissons de la rivière, dont on note le comportement. On le fait plus fréquemment a posteriori, au Laboratoire, sur des échantillons d'eau prélevés en rivière et préalablement analysés chimiquement.

La comparaison des résultats de l'analyse chimique qui met en évidence les produits suspects (composés ammoniacaux, sulfures, produits phénoliques, etc...) qu'elle peut contenir et dont on connaît la toxicité par cles travaux de laboratoire, avec ceux donnés par le test biologique au Laboratoire permet de donner des conclu- 
sions mieux établies. Le test sur le terrain est évidemment le plus sûr, celui qui entraine le plus facilement la conviction des juges. Cependant, il exige la présence de poissons sains des mêmes espèces que ceux qui peuplent la rivière et il n'est pas toujours commode et rapide de se munir de poissons en bon état. L'analyse chimique accompagnée du test biologique au Laboratoire a posteriori n'est pas évidemment sans critique, puisque entre le moment du prélèvement des échantillons en rivière et celui de l'analyse au laboratoire, il s'écoule un délai qui est susceptible d'altérer l'eau, surtout si le temps est chaud. Pour limiter au maximum ces inconvénients, les - échantillons sont placés, dès leur arrivée au Laboratoire, dans un frigidaire.

Ces pollutions accidentelles aiguës qui se traduisent par des mortalités de poissons et qui sont les plus spectaculaires ne sont cependant pas les plus dangereuses. Les pollutions chroniques, larvées, insidieuses qui, peu à peu, empêchent le poisson de se nourrir et de se reproduire ou qui même plus simplement altèrent la santé des cours d'eau et des lacs sans nuire aux poissons, sont beaucoup plus redoutables car l'action de l'Homme est ici limitée, le mal une fois fait.

A moins de supprimer les usines riveraines, ce qui est inconcevable, il est impossible maintenant de corriger des rivières telles l'Ondaine et le Furans, sur la Loire, telles celles de la région du Nord où toute vie animale et végétale est absente.

Ce sont là évidemment des cas extrêmes et nos cours d'eau sont moins atteints, mais pour ne citer que la Seine, les Parisiens se souviennent des hécatombes massives de poissons qui se produisirent brusquement dans les étés I954 et I958 et qui nuisirent si fortement à l'esthétique de la Capitale qu'on dut en faire disparaître clandestinement, la nuit, les cadavres, des anses où ils s'étaient accumulés. Sous la double action de la température et de la baisse des eaux, la pollution chronique s'était transformée en pollution aiguë.

Les pollutions peuvent se faire sentir sur 30,40 , voire même $5^{\circ}$ kilomètres.

Nos cours d'eau, surtout s'ils ont un certain courant, se défendent cependant plus facilement que nos lacs, car ils sont capables, sous l'action de l'oxygène, d'autoépurer plus ou moins bien, suivant les cas, les eaux organiques qu'ils reçoivent. Mais nos lacs sont moins bien partagés. Les matières organiques qui s'accumulent à la longue dans les fonds créent un milieu réducteur, impropre à la vie des poissons de fond, l'Omble-Chevalier, par exemple, si apprécié en Savoie; elles développent en outre des quantités prodigieuses de certaines Algues qui forment ce que l'on appelle " des fleurs d'eau ». Ces Algues, souvent dépourvues de Chlorophylle, sont alors incapables d'oxygéniser suffisamment le milieu, puis, par 
l'amoncellement de leurs cadavres sur le fond, elles finissent par le rendre putride. La situation est irréversible. Il y a encore des poissons, mais la couleur de ces lacs malades est peu engageante. Ils prennent, en certaines périodes, une couleur " rouge sang ) due à la Cyanophycée Oscillatoria rubescens que les Suisses' qui l'ont observée pour la première fois il y a une centaine d'années dans le Lac de Morat, nomment du nom imagé de " sang des Bourguignons ) (Burgunder Blut), en souvenir de la célèbre bataille de ce nom, sous Charles le Téméraire (I476). C'est le cas notamment du Lac de Nantua et la ville, je le sais personnellement, se préoccupe activement en ce moment de cette " maladie ), si préjudiciable au tourisme. Le Léman, le Lac du Bourget, le Lac d'Annecy, bien que moins atteints, en raison de leur grande profondeur, ne sont pas cependant en bon état et leur évolution dangereuse est rapide.

Sans aller très loin, une belle rivière de la région de Paris, la Blaise, près de Dreux, qui naît dans un étang souillé depuis quelques années par le camp américain voisin, est désagréablement colorée de brun par une fleur d'eau due à une algue provoquée par l'accumulation de matières organiques dans l'étang. La station d'épuration qui a été installée, mais trop tard, et qui fonctionne bien. est incapable à elle seule de rétablir la situation.

Une Administration, comme celle que j'ai l'honneur de représenter ici, ne pouvait se désintéresser de sa double mission, de protection de la nature et de défense de la pêche fluviale dans les rivières ni navigables ni flottables.

Le problème de la protection des eaux contre la pollution est un problème à la fois technique, économique et social. Il est technique par l'examen qu'il convient de faire des procédés possibles d'amélioration des eaux. ćconomique par le coût de ces procédés, et social par sa répercussion sur la pêche et le tourismé.

Le problème technique, reconnaissons-le objectivement, est parfois difficile à résoudre. Il est parfaitement résolu pour l'épuration des eaux urbaines, peu chargées en matières organiques (lits bactériens, boues activées); il l'est également pour certaines eaux résiduaires industrielles, par exemple pour les eaux acides de décapage. Mais il ne l'est pas pour toutes les industries.

Sans être parfaits, certains procédés d'utilisation gćnérale sont tout de même susceptibles d'apporter une amélioration sensible à la qualité des eaux résiduaires, par exemple les procédés par floculation' qui permettent la précipitation des matières solides en suspension dans l'eau, ne laissant subsister que les seules matières dissoutes, ce qui diminue d'autant leur nocuité. 
Les procédés par aspersion et spécialement ceux par aspersion fine qui consistent à arroser les champs d'épandage à l'aide du flot résiduaire, donnent des résultats excellents pour les eaux organiques, comme celles des distilleries, sucreries, féculeries, laiteries. Elles supposent toutefois la présence de champs à proximité de l'usine.

Si, dans certains cas, l'industriel ne peut techniquement utiliser un procédé idoine, il lui est toujours possible alors d'adopter une “ politique de l'eau à l'usine ) c'est-à-dire une " économie de l'eau ), lui permettant par un aménagement approprié de limiter les dégâts: échelonnement des déversements dans le temps et en fonction du débit de la rivière réceptrice, neutralisation des eaux acides par les eaux alcalines, recyclage, etc...

Le point de vue économique est naturellement celui qui préoccupe le plus les industriels, car les procédés d'épuration sont souvent coûteux. "Vous allez augmenter notre prix de revient », disent-ils d'ordinaire. C'est là un argumenut de poids évidemment. Tout dépend d'ailleurs de l'importance de l'usine; certains industriels que nous connaissons n'ont pas craint de consacrer des sommes importantes à l'épuration de leurs eaux: plusieurs centaines de millions parfois.

Il est évidemment plus difficile aux petites usines de faire un effort proportionnellement aussi important. Mais trop d'entre elles n'essaient-elles pas — c'est humain — de jeter le manche avant la cognée?

Quoi qu'il en soit, il semble que l'Etat devrait leur accorder une aide pour les encourager dans un effort éminemment souhaitable. Nous en reparlerons tout à l'heure.

Le problème de la pollution est enfin un problème social. Non seulement la pollution incommode les riverains et les baigneurs comme ce fut le cas, par exemple, pour la petite cité de la Seguinière près de Cholet, comme ce fut le cas des Parisiens en aval de la Capitale, mais elle nuit encore au tourisme comme je l'ai signalé tout à l'heure pour la ville de Nantua et comme il en est depuis cles années pour la ville de Dax, l'Adour chariant des « tampons ") d'aspect et de couleur peu engageants dus à des Champignons et Bactéries filamenteuses (Leptomitus, Sphaerotilus) causés par la pollution d'une importante usine de pâte à papier, située à quelques dizaines de kilomètres en amont.

Mais beaucoup plus grave encore, au point de vue social, est le domnage causé à la pêche sportive qui a, en France, une importance réelle puisqu'elle intéresse près de trois millions d'homme ou de femmes, la plupart de conditions modestes. Ces pêcheurs occasionnels, vivant en ville pour le plus grand nombre et souvent dans 
les cités industrielles où l'atmosphère est viciée, sont heureux de passer leurs loisirs du dimanche, en famille, au bord de l'eau. Ils souffrent réellement de ne pas trouver à proximité de leur demeure le moyen d'une passe-temps agréable et sain. Ils paient, d'autre part, depuis 194I, une taxe d'Etat, en dehors de leur cotisation obligatoire à une société de pêche et à la Fédération départementale. Ils sont en droit de pouvoir pêcher en toute tranquillité.

Pour éviter tout ennui social, les industriels sont tentés trop souvent de recourir à une méthode commode mais que je dois dénoncer ici, car elle ne saurait régler en aucune façon le problème qui nous préoccupe. Ils versent chaque année à la Société de pêche locale une indemnité sous prétexte de repeuplements pour réparation du préjudice causé par une pollution chronique. Cette indemnité ne peut servir utilement car si le milieu est transformé biologiquement, comme je l'ai montré tout à l'heure, ce qui est le cas général, les repeuplements ne peuvent être efficaces. Souvent d'ailleurs, ils n'ont pas lieu et les indemnités servent à quelques banquets, pendant que la pollution continue à altérer gravement la rivière.

L'Etat intervient par des mesures réglementaires et de police et par des mesures de contrôle.

Les mesures de police résultent de deux législations parallèles, l'une concernant la pêche, l'autre concernant les établissements industriels insalubres.

La loi du I5 avril I829, en son article 25, reprenant d'ailleurs presque mot pour mot l'ordonnance des Eaux et Forêts de Colbert de I669 (I) interdit le rejet en rivière de " drogues ou appâts " susceptibles de " tuer ou énivrer le poisson ». De toute évidence, seuls les délits de braconnage étaient visés dans une loi datant de la naissance de l'industrie; les sanctions étaient sévères: amendes et même peine de prison. Jusqu'en 1949, cette loi a permis cependant, par extension admise en jurisprudence, de réprimer les pollutions industrielles les plus marquantes. Je ne crois pas d'ailleurs qu'aucun Directeur d'usine ait été jamais condamné à une peine de prison ferme; on ne pouvait tout de même pas assimiler un industriel négligent à un braconnier.

Cette loi a été modifiée à l'Assemblée Nationale, par initiative parlementaire sans débat et sans consultation préalable de l'Administration, le 9 avril 1949 .

Le nouveau texte distinguait pour la première fois la pollution industrielle de la pollution de braconnage, mais subordonnait la

(1) Art. I4 de l'Ordonnance de I669 sur leś Eaux et Forêts :

"Défendons à toutes personnes de jeter dans les rivières: noix vomique, coque du Levant, mommie et autres drogues ou appâts, à peine de punition corporelle $\gg$. 
transaction administrative à l'avis préalable de la Fédération départementale de pêche, ce qui provoqua de vives réclamations des milieux industriels. La loi ne prévoit aucune condition ni pour l'effuent ni pour la rivière.

Parallèlement à cette législation et sans aucun lien avec elle, la loi du ro décembre rgr7 sur les établissements insalubres, en fixant les règles administratives que doivent suivre les industriels avant d'établir une usine douteuse au bord d'un cours d'eau, énumère de son côté des sanctions pénales, mais d'amendes seulement.

Une très intéressante instruction du 6 juin I953 émanant du Ministère de l'Industrie et du Commerce fixe les normes que doivent avoir les effluents industriels suivant leur situation: rejet dans un réseau public d'assainissement pourvu d'une station d'épuration collective; rejet dans un milieu naturel peu pollué, moyennement pollué, ou très pollué; rejet dans une rivière à proximité de prises d'eau pour les villes, de plages, de bancs de coquillages ou de réserves à Salmonidés.

$\mathrm{Si}$ utiles que soient ces normes, elles ne peuvent avoir en fait qu'une valeur relative, la pollution dépendant avant tout du débit relatif de l'effluent et de la rivière. Telle usine polluera un petit cours d'eau et ne polluera pas une grande rivière. Il serait donc préférable de fixer des normes pour la rivière elle-même ou plus exactement une charge de pollution admissible, mais on se heurte alors pour cette clétermination à de sérieuses difficultés pratiques que nos amis belges connaissent bien.

Il était évidemment paradoxal de ne pas jeter un pont. d'une part entre ces deux législations et, d'autre part, de ne pas rendre à l'Etat son pouvoir d'arbitre gravement compromis par l'avis préalable obligatoire des Fédérations de pêche. Ce fut l'objet de l'Ordonnance du 3 janvier I959, qui prévoit, avant toute transaction, l'avis de l'Inspecteur départemental des Etablissements classés pour les entreprises relevant de la loi précitée du I9 décembre I9I7, en supprimant par contre, l'avis obligatoire des Fédérations de Pêche, celles-ci ayant toujours possibilité de se porter parties civiles pour réparation de dommages.

A côté de ces mesures réglementaires et de police, l'Etat devait envisager parallèlement des mesures techniques, préventives puisque. d'après les exemples que nous avons cités tout à l'heure, il vaut mieux en matière de pollution " prévenir que guérir ), si tant est même qu'il soit possible souvent de guérir, lorsque l'intervention est trop tardive.

Ces mesures techniques, encore à l'état d'ébauche, ont été l'œuvre de la Station Centrale d'Hydrobiologie appliquée avec la précieuse aide financière du Conseil Supérieur de la Pêche, organe para-admi- 
nistratif présiclé par le Directeur Général des Eaux et Forêts, chargé cle défninir la politique française en matière de pêche fluviale et de répartir au mieux le montant de la taxe piscicole instituée en I94I.

Le Laboratoire de chimie des eaux de la Station Centrale d'Hydrobiologie appliquée étudie chaque année au moyen de l'analyse chimique du liquide pollué et du test biologique sur le poisson, près de 500 pollutions différentes représentant i 500 analyses et autant de tests biologiques; d'autre part, 8 camionnettes aménagées en laboratoires ambulants et réparties dans les principales villes de France visitent et contrôlent régulièrement un grand nombre d'usines. Le but de ce service n'est pas répressif ; il veut, avant tout, aider les industriels, leur montrer par des analyses effectuées devant eux la mauvaise qualité de leurs eaux et leur indiquer officieusement (car ce n'est pas le rôle de l'Administration d'imposer tel système d'épuration plutôt que tel autre) ce qu'ils peuvent faire pour améliorer leurs déversements résiduaires. Depuis la création de ce contrôle en I949, les eaux industrielles de plus de 3000 usines différentes ont été ainsi analysées et contrôlēes, généralement plusieurs fois ; un fichier par département, par cours d'eau et par nature d'industrie permet de suivre, pour chacune d'elles, l'évolution de la pollution. Sans doute, c'est encore un Service insuffisant qu'il faudrait développer mais dont l'efficacité est certaine. Il a créé entre les Industriels et l'Administration un climat de confiance qui n'existait pas jusqu'à ce jour.

Il reste cependant beaucoup à faire pour assurer une protection efficace de nos rivières et de nos lacs.

I1 semble tout d'abord qu'un organisme coordinateur, ểmanant par exemple directement de la Présidence dı Conseil, serait des plus utiles, eu égard à l'extrême variété des administrations qui ont à connaître des problèmes de la pollution et dont les vues, forcément différentes, auraient intérêt à être uniformisées. Ces administrations sont les suivantes et $j$ 'en oublie peut-être:

Ministère de la Santé Publique et de la Population: Baignades.

Ministère de l'Industrie et du Commerce: Législation des établissements insalubres.

Ministère de l'Agriculture:

Direction Générale des Eaux et Forêts: Police de la pêche dans les seules rivières non canalisées - Pisciculture dans l'ensemble du territoire.

Direction Générale du Génie Rural et de l'Hydraulique Agricole: Eaux non domaniales.

Ministère des Travaux Publics, du Transport et du Tourisme: Police de la pêche dans les canaux et rivières canalisées.

Ministère de la Construction: projets de déversements à l'égout. 
En dehors de la coorlination des deux législations, celle de la pềche et celle des Etablissements Classés, effective depuis l'Ordonnance du 3 janvier I959, il n'y a guère eu jusqu'à ce jour que 1'heureuse collaboration prévue sur le plan départemental par la circulaire du Ministère de l'Industrie en clate du ro juin I948, au sein d'une Commission de pollution où figurent les représentants des administrations intéressées.

Ce sont là d'heureuses initiatives qu'il conviendrait de développer.

Il appartiendrait cependant à l'Etat d'envisager, semble-t-il, d'autres mesures. Tout d'abord la multiplication désirable de Commissions de bassins fluviaux chargées d'étudier pour une rivière donnée les répercussions de la pollution et les moyens d'y remédier en commun, commissions qui ont donné d'excellents résultats dans la Chiers, dans l'Orne, dans la Meurthe; le bassin est, en effet, une unité géographique. Les mesures suivantes seraient certainement très utiles:

I ${ }^{\circ}$ L'extension des procédés d'épandage par aspersion se trouve limitée par l'impossibilité pour l'industriel d'acquérir les champs d'épandage désirables; il faudrait envisager une procédure d'expropriation pour cause d'utilité publique. La législation actuelle ne le permet pas.

$2^{\circ}$ Certaines rivières spécialement intéressantes pour le tourisme et pour la pêche pourraient être " réservées »: interdiction serait faite d'y installer une usine sur les bords. D'autres cours d'eau, par contre, déjà très pollués, pourraient être abandonnés à l'industrie, sans grand inconvénient.

$3^{\circ}$ Des subventions devraient être accordées plus facilement par l'Etat aux collectivités publiques désireuses d'épurer leurs eaux. Le coût de ces installations très efficaces est élevé et certaines villes éprouvent de ce fait de la difficulté. L'amélioration qui résulterait cependant de leur multiplication serait appréciable non seulement par la suppression de la pollution organique par les égouts, mais encore par la possibilité ainsi offerte aux eaux industrielles d'être diluées et, si elles sont organiques, épurées avant rejet dans la rivière. A condition que la densité industrielle ne soit pas excessive eu égard à la densité urbaine, ce qui est, en France, le cas général, i) n'y a aucun inconvénient à redouter de cette addition pour le bon fonctionnement des stations.

$4^{\circ}$ Pourquoi, enfin, l'Etat n'accorderait-il pas une aide financière aux industriels soucieux de la qualité de leurs eaux résiduaires, ainsi que cela se fait dans certains Länder allemands, par exemple, au moyen de dégrèvements d'impôts? 
La protection des rivières déborde le cadre d'un Etat: elle pose, en effet, pour les cours d'eau traversant une frontière, des problèmes délicats, 1'Etat situé en aval recevant généralement les eaux polluées de son voisin.

En ce qui concerne la France, il existe pour les régler des commissions internationales, telles que celles de la Sambre, de la Sarre et notamment celle de la protection du Rhin contre la pollution qui groupe les 5 Etats riverains de ce fleuve. A la suite d'une réunion d'experts internationaux, au printemps I957, la Commission économique pour l'Europe à Genève, a créé un bureau de liaison avec d'autres organismes internationaux intéressés, l'Organisation mondiale de la Santé et la F.A.O. (Division des pêches). Ce n'est encore qu'un début; mais la coopération internationale doit se développer dans ce domaine tout à fait à l'ordre du jour.

Cependant, pour que des initiatives d'ordre administratif et réglementaire aient quelques chances d'être effiçaces, il faudrait au préalable créer un climat favorable, le problème de la pollution ne pouvant être réglé qu'en accord avec tous les intéressés. Il serait donc hautement souhaitable que puisse se fonder en France une Ligue pour la protection des eaux comme il s'en est constitué une, après Guerre, en Allemagne et en Suisse. Il s'agit là d'une initiative essentiellement privée qui grouperait tous ceux qui s'intéressent à l'eau, industriels, pêcheurs, techniciens des procédés d'épuration, directeurs de laboratoire et simples amateurs de la Nature. Par son action de propagande clans les Ecoles, auprès des collectivités, par les contacts qu'elle provoquerait entre les industriels et les usagers de l'eau, par l'appui qu'elle apporterait aux initiatives gouvernementales, elle permettrait de trouver plus aisément la solution à des problèmes difficiles et pourtant urgents, qui commencent seulement à être abordés efficacement.

Malgré les difficultés qui restent à résoudre, il doit être partout possible, avec un esprit de compréhension réciproque et en usant plus de la persuasion que de la coercition, de faire vivre côte à côte pour le plus grand bien d'un pays, une industrie active au bord d'une rivière, saus pour cela compromettre sa beauté, sa salubrité et les joies paisibles de la pêche qui sont celles de 3000 ooo de Français.

P. ViVier. 